\title{
Time-integrated supernova neutrino flux from a nearby cluster
}

\author{
Van T. Nguyen ${ }^{1}$ and Calvin W. Johnson * \\ Department of Physics, San Diego State University, 5500 Campanile Drive, San \\ Diego CA 92182-1233, USA
}

\begin{abstract}
The rate of gravitational collapse (type II) supernovae in our galaxy is uncertain by a factor of three or more. One way to determine the galactic supernova rate is through a radiogeochemical experiment (for example, the molybdenum-technetium experiment) that would integrate the neutrino flux over several million years. While such a measurement is designed to integrate the flux over the entire galaxy, nearby star-forming regions could skew the results. We model the flux from a recently identified such region, the Scorpius-Centaurus OB association, and compare with the flux from the rest of the galaxy.
\end{abstract}

Key words: Supernovae, Neutrinos, Radiogeochemical experiments PACS: 95.55.Vj, 97.60.Bw, 98.20.Af

\section{Introduction}

Type II supernovae from the gravitational collapse of massive, short-lived stars are the engines that drive the chemical evolution of galaxies. While the basic plot of a gravitational collapse supernova (SN) is known[1], the details are murky: it is difficult to obtain a successful explosion in a computer model, and it is now generally believed that one must treat the turbulent hydrodynamics and/or the neutrino transport with great care in order to produce an outward explosion. The hot, neutrino-driven neutron wind in the aftermath of a gravitational collapse $\mathrm{SN}$ is a prime candidate for $r$-process nucleosynthesis,

\footnotetext{
* Corresponding author

1 Current address: Columbia University, Department of Physics, 538 W. 120th St New York, NY 10027
} 
but even that chapter is not finished, inasmuch as it depends sensitively on entropy, lepton fractions, and neutrino physics[2].

The famous supernova 1987A [3] in the Large Magellenic Cloud outside our galaxy (distance $\sim 50 \mathrm{kpc}$ ) provided us with slightly less than two dozen neutrino events; with the current generation of neutrino observatories, should a gravitational collapse SN occur within our galaxy, we will harvest a wealth of information to help diagnose the physics of gravitational collapse and subsequent explosion (and perhaps neutrinos themselves).

Unfortunately, at least for science, galactic SN events are rare. Various arguments generally suggest the rate of Type II galactic SNe to be between 1 and 3 per century[4], although some earlier estimates were as high as 10 per century[5]. A galactic SN has not been visually observed for nearly four centuries, although we can only visually observe $\sim 1 / 6$ of our galaxy; in the $18+$ years since SN 1987A, neutrino observatories have failed to detect any galactic SN, which should produce hundreds or thousands of events. Knowledge of the galactic SN rate would help plan for neutrino observatories.

Because the interval between galactic $\mathrm{SNe}$ is decades, an alternate approach to determining the rate is a radiogeochemical experiment[6,7]. A radiogeochemical experiment is a generalization of radiochemical experiments such as the chlorine-argon and gallium-germanium experiments[8], but instead of a human-made target, the parent nuclei are in a naturally buried ore body and the daughter is a long-lived but very rare isotope. The daughter must be produced primarily through neutrino interactions (hence one must be able to limit production through fission, cosmic rays, etc.), with a half-life short enough so no primordial atoms remain but long enough to "collect" sufficient data. As discussed below, and elsewhere, it is possible to identify plausible scenarios that will integrate the flux of neutrinos from galactic SNe.

Gravitational collapse SNe occur only in massive $\left(M>8 M_{\text {solar }}\right)$ stars with short lives (typically 10-50 My), so they are associated with star-forming regions. If one uses a smooth, exponential parameterization for the disk portion of the Milky Way[9] one finds a flux equivalent to a point source $4.6 \mathrm{kpc}$ distant from Earth[7]. But this ignores nearby variations in the SN distribution, which, weighted by the inverse of the square of the distance, could have a disproportionate effect.

In this paper we consider the effect of a nearby star-forming region, the Scorpius-Centaurus OB association (Sco-Cen), which is thought to have had 20 SNs over a period of roughly 10 My approximately 100-130 pc from Earth[10]. In order to understand the effect of the SN neutrino flux from this star-forming region, we consider the time-dependence of the distance to the cluster, through proper motion, and the finite width of the clusters. The focus of this paper 
is on the relative contributions from nearby clusters, Sco-Cen in particular, relative to the neutrino flux from SNe throughout the galaxy.

\section{The Mo-Tc experiment}

The example we consider is the molybdenum-technetium (Mo-Tc) experiment [6,11]; other potential radiogeochemical experiments are discussed in Ref. [7]. The Mo-Tc experiment was originally proposed to measure the long-term solar neutrino flux, in order to test the hypothesis that the measured low flux of ${ }^{8} \mathrm{~B}$ solar neutrinos might be caused by nonstandard solar models. Such nonstandard solar models have been ruled out through helioseismology and detection of neutrino flavor oscillations [12]. Inverse beta decay induced by neutrino flux on molybdenum ore produces technetium

$$
{ }^{98} \mathrm{Mo}+\nu \rightarrow{ }^{98} \mathrm{Tc}+e^{-}
$$

${ }^{98} \mathrm{Tc}$ has a half-life of $4.2 \mathrm{My}$, short enough so that it cannot be primordial in origin, but long enough to integrate over the mixing time of the solar core.

Originally the main source of neutrinos considered were ${ }^{8} \mathrm{~B}$ neutrinos from the Sun. It was later realized[7] that ${ }^{97} \mathrm{Tc}$, with a half-life of $2.6 \mathrm{My}$, could serve as a tracer of supernova neutrinos through the reaction

$$
{ }^{98} \mathrm{Mo}+\nu \rightarrow{ }^{97} \mathrm{Tc}+n+e^{-} .
$$

Solar neutrinos are generally too low in energy, however, to knock-out a neutron and produce ${ }^{97} \mathrm{Tc}$ through (2), which has a threshold of $7.28 \mathrm{MeV}$, but more energetic SN neutrinos can easily do so. Table 1 gives the relevant crosssections as computed in [7]. (In this paper we do not consider neutrino flavor oscillations; such oscillations will certainly change cross-sections[13], but focus only on relative contributions to supernova fluxes.)

The larger cross-sections of SN neutrinos allow them to compete with solar neutrinos as a source of ${ }^{97} \mathrm{Tc}$ despite having a lower flux. The standard solar model flux of ${ }^{8} \mathrm{~B}$ solar neutrinos is $\sim 6 \times 10^{6} \mathrm{~cm}^{-2} \mathrm{~s}^{-1}$ [8] (this does not include neutrino flavor oscillations, which will reduced this considerably), while the flux of galactic SN neutrinos is much smaller, about

$$
R \times N_{58} \times 1.3 \times 10^{3} \mathrm{~cm}^{-2} \mathrm{~s}^{-1}
$$

where $R$ is the rate of galactic gravitational collapse SNe per century, somewhere around 1 to 3 , and $N_{58}$ is the number of neutrinos emitted per collapse 
in units of $10^{58}$, about 0.45 for electron neutrinos. (A similar study [14] looks at the neutrino flux from thermonuclear burning throughout the galaxy and from extragalactic sources.)

This beautiful dichotomy is spoiled somewhat by the existence of ${ }^{97} \mathrm{Mo}(9.55 \%$ compared to $24.13 \%$ for ${ }^{98} \mathrm{Mo}$ ), so that

$$
{ }^{97} \mathrm{Mo}+\nu_{\mathrm{solar}} \rightarrow{ }^{97} \mathrm{Tc}+e^{-}
$$

can also contribute. The cross-section is not known because there is no experimental data on the Gamow-Teller strengths, but a reasonable scaling argument [7] can be used to derive the ${ }^{97}$ Mo cross-sections in Table 1; one would need to subtract off the solar contribution in order to measure the galactic SN rate. (An attempt to mount the Mo-Tc experiment was begun by K. Wolfsberg and collaborators in the 1980's, but the original experiment was never completed due to funding limitations [15]. We note, however, that the Henderson molybdenum mine in Colorado, the site of the original Mo-Tc experiment, is still active and is at the time of this writing a candidate for siting the National Underground Science Laboratory, and it is not impossible that the Mo-Tc experiment could be resurrected.)

\section{Time-dependence and nearby events}

Sco-Cen can be divided into through subgroups: Lower Centarus Crux (LCC), Upper Centaurus Lupus (UCL), and Upper Scorpius (US), each of whose distance from the Earth varied between 100 and 240 pc over the past 10 $\mathrm{My}[16,10]$ due to proper motion. Each of the subgroups have a finite window over which SNe could have occurred: 3 My for US, 7 My for LCC, and $10 \mathrm{My}$ for UCL. The SN rate in each subgroup is assumed to be approximately 1 per $\operatorname{My}[16,10]$.

In order to account for the time-dependence of the neutrino flux, we note we must solve

$$
\frac{d N}{d t}=-\lambda N(t)+\sigma \Phi(t)
$$

where $N(t)$ is the number of daughter nuclei, here ${ }^{97} \mathrm{Tc}, \lambda$ is the decay constant, $\sigma$ is the reaction cross-section, and $\Phi(t)$ is the time-dependent flux. From the data in Ref. [10] we approximated $\Phi$ as a quadratic in $t$; this can be solved analytically. We assumed the rate of SNe is constant in time.

The most useful way to present the results is to compute an effective distance 
$r_{\text {eff }}$; the effect of the time-integrated flux for the cluster in motion is the same as a static cluster fixed at the distance $r_{\text {eff }}$.

We found that the effective distance $r_{\text {eff }}$ can be approximated by the following simple prescription:

$$
\frac{1}{4 \pi r_{\mathrm{eff}}^{2}}=\langle\Phi\rangle=\frac{\int_{-T}^{0} \Phi(t) e^{-\lambda t} d t}{\int_{-T}^{0} e^{-\lambda t} d t}
$$

where $T$ is the time before the present that SNe began in the subgroup; this formulation, which is simpler than solving Eq. (5), agrees with such solutions to within a fraction of a percent. Table II gives the average fluxes and $r_{\text {eff }}$ for each subgroup studied. The total average flux from Sco-Cen is then the sum of the fluxes in the middle column, $450 \mathrm{~cm}^{-2} \mathrm{~s}^{-1}$.

\section{Finite-size corrections and extra-near events}

We also considered the fact that the star-forming subgroups have a finite width, on the order of 20-30 pc. By considering a sphere of uniform density with a center at a distance $r$ from Earth and with rms radius $\sigma$, we find that the flux from the cluster is

$$
\Phi=\frac{1}{4 \pi r^{2}}\left(1+\frac{\sigma^{2}}{3 r^{2}}\right) .
$$

Monte Carlo simulations with a Gaussian distribution but the same rms radius agreed with this correction term. Thus the correction due to finite width is relatively small.

We further took the opportunity of our Monte Carlo simulation to investigate the question of extra-near SN events. Anomalous deposits of ${ }^{60} \mathrm{Fe}$ in deep-sea crust have been linked to a nearby (a few 10 pc) SN within the last few My $[17,18]$. Benítez, Maíz-Apellániz, and Canelles [10] suggest this anomaly could be explained by a SN in Sco-Cen; taking a cluster at 100 pc with a Gaussian with an rms width of 25-30 pc, they argue for a SN as close as 40 pc from Earth ( $2 \sigma$ lower limit). Unfortunately this argument is based upon a one-dimensional Gaussian distribution and is wrong for a three-dimensional distribution. In our Monte Carlo simulations, we found relatively few simulation runs that placed a SN at about 40 pc; in fact only a fraction of a percent of any given simulation with 20 SNe gives an event closer than 50 pc. Increasing the rms width of the cluster to $40 \mathrm{pc}$ still only yields a near-Earth ( $<50 \mathrm{pc}$ ) event only $12 \%$ of

the time. Of course, such simulations depend sensitively on the tail of the 
distribution, which might be non-Gaussian. Nonetheless, while Sco-Cen is not ruled out as a candidate source for the ${ }^{60} \mathrm{Fe}$ anomaly, the statistical linkage is weaker than previously argued. Conversely, one could possibly use ${ }^{60} \mathrm{Fe}$ and other radioisotopes to constrain nearby events [19] and sharpen our modeling of the flux from such events.

\section{Conclusions}

We have considered the potential effect a nearby star-forming region, Sco-Cen, would have on radiogeochemical measurements of the galactic supernova flux, in particular the Mo-Tc experiment. Although we performed detailed calculations, we found that both the time-dependence of the flux due to proper motion and the finite width of a region can be calculated through simple, effective prescriptions which could be applied to any cluster and any radiogeochemical experiment of interest. The flux from Sco-Cen is roughly the same as that from throughout the galaxy if the galactic SN rate is 1 per century; this "background" could limit the ability of a radiogeochemical experiment to measure the overall rate. At higher galactic rates the Sco-Cen contribution will be relatively small.

\section{Acknowledgements}

The U.S. Department of Energy supported this investigation through grant DE-FG02-96ER40985. VTN gratefully acknowledges support from the SDSU

McNair Scholars program and from NIH SDSU MARC grant \#5 T34 GM08303.

\section{References}

[1] S.E. Woosely, A. Heger, and T. A. Weaver, Rev. Mod. Phys. 74, (2002) 1015; S.E. Woosely and T.A. Weaver, Ann. Rev. Astro. Astrophys. 24, (1986) 205.

[2] B. S. Meyer, Ann. Rev. Astro. Astrophys. 32, (1994) 153.

[3] W. D. Arnett, J. N. Bahcall, R. P. Kirshner, and S. E. Woosley, Ann. Rev. Astro. Astrophys. 27, (1989) 629.

[4] S. van den Berg and G. A. Tammann, Ann. Rev. Astro. Astrophys. 29, (1986) 363.

[5] J. N. Bahcall and T. Piran, Astrophys. J. 267, L77 (1983). 
[6] G. A. Cowan and W. C. Haxton, Science 216, 51 (1982).

[7] W. C. Haxton and C. W. Johnson, Nature 333, 325 (1988).

[8] , J. N. Bahcall, Neutrino Astrophysics (Cambridge University Press, Cambridge 1989).

[9] J. N. Bahcall and R. M. Soneira, Ap. J. Suppl. 44, 73 (1980).

[10] N. Benítez, J. Maíz-Apellániz, and M. Canelles, Phys. Rev. Lett. 88, 081101 (2002).

[11] K. Wolfsberg, et al., in Solar Neutrinos and Neutrino Astronomy, M. L. Cherry, W. A. Fowler and K. Lande, eds, 196 (American Institute of Physics, New York, 1982).

[12] J. N. Bahcall and R. K Ulrich, Rev. Mod. Phys. 60, 297 (1988); Q. R. Ahmad, R. C. Allen, T. C. Andersen, J. D. Anglin, et al., Phys. Rev. Lett. 87, 071301 (2001); S. Turck-Chièze, S. Couvidat, L. Piau, J. Ferguson, et al., Phys. Rev. Lett. 93, 211102 (2004).

[13] C. W. Johnson, in Trends in Astroparticle Physics, D. Cline and R. Peccei, editors (World Scientific, Singapore, 1992) 526.

[14] C. Porciani, S. Petroni, and G. Fiorentini, Astropart. Phys. 20, 683 (2004).

[15] W. C. Haxton, private communication.

[16] J. Maíz-Apellániz, Ap. J. 560, L83 (2001).

[17] K. Knie, G. Korschinek, T. Faestermann, C. Wallner, et al, Phys. Rev. Lett. 83, 18 (1999).

[18] K. Knie, G. Korschinek, T. Faestermann, E. A. Dorfi, et al, Phys. Rev. Lett. 93, 171103 (2004).

[19] B. D. Fields, K. A. Hochmuth, and J. R. Ellis, Astrophys. J. 612, 902 (2005). 
Table 1

Neutrino capture cross-sections for various sources; taken from [7]

\begin{tabular}{lll} 
Reaction & $\nu$ source & $\sigma\left(10^{-42} \mathrm{~cm}^{2}\right)$ \\
\hline${ }^{98} \mathrm{Mo}\left(\nu, e^{-}\right){ }^{98} \mathrm{Tc}$ & Solar ${ }^{8} \mathrm{~B}$ & 3 \\
${ }^{98} \mathrm{Mo}\left(\nu, e^{-}\right)^{98} \mathrm{Tc}$ & $\mathrm{SN}$ & 50 \\
${ }^{98} \mathrm{Mo}\left(\nu, e^{-}\right)^{97} \mathrm{Tc}+n$ & Solar ${ }^{8} \mathrm{~B}$ & 0.3 \\
${ }^{98} \mathrm{Mo}\left(\nu, e^{-}\right)^{97} \mathrm{Tc}+n$ & $\mathrm{SN}$ & 150 \\
${ }^{97} \mathrm{Mo}\left(\nu, e^{-}\right){ }^{97} \mathrm{Tc}$ & Solar ${ }^{8} \mathrm{~B}$ & 1.2 \\
${ }^{97} \mathrm{Mo}\left(\nu, e^{-}\right)^{97} \mathrm{Tc}$ & $\mathrm{SN}$ & 18
\end{tabular}

Table 2

Subgroups in Sco-Cen

\begin{tabular}{cccc} 
Subgroup & $\mathrm{T}(\mathrm{My})$ & $\langle\Phi\rangle\left(\mathrm{cm}^{-2} \mathrm{~s}^{-1}\right)$ & $r_{\mathrm{eff}}(\mathrm{pc})$ \\
\hline US & 3 & 111 & 154 \\
LCC & 7 & 218 & 110 \\
UCL & 10 & 120 & 148
\end{tabular}

